\title{
Visible and near-infrared backscattering spectroscopy for sizing spherical microparticles
}

\author{
Lucía B. Scaffardi, Fabián A. Videla, and Daniel C. Schinca
}

\begin{abstract}
Scattering is a useful tool for the determination of particle size in solution. In particular, spectroscopic analysis of backscattering renders the possibility of a simplified experimental setup and direct data processing using Mie theory. We show that a simple technique based on near-infrared (NIR) backscattering spectroscopy together with the development of the corresponding algorithm based on Fourier transform (FT) and Mie theory are a powerful tool for sizing microparticles in the range from 8 to $60 \mu \mathrm{m}$ diameter. There are three wavelength intervals in the NIR, within which different diameter ranges were analyzed. In each one, the FT yields a coarse diameter value with an uncertainty dependent on the wavelength range. A more accurate value is obtained by further applying cross correlation between experimental and theoretical spectra. This latter step reduces the uncertainty in diameter determination between $30 \%$ and $40 \%$, depending on wavelength interval and particle diameter. These results extend previous information on visible backscattering spectroscopy applied to sizing microparticles in the range between 1 and $24 \mu \mathrm{m}$ diameter. This technique could be the basis for the construction of a portable and practical instrument. (C) 2007 Optical Society of America

OCIS codes: $\quad 300.0300,290.1350,300.6340,120.5820$.
\end{abstract}

\section{Introduction}

Size characterization of particulate material in solution is important for quality control processes in pharmacological, paint, cement, cosmetic, and pesticide industries, among others. Different optical techniques based on light scattering have been developed and applied to sizing particles due to their ease of use and noninvasive nature. Some are based on measurements of angular scattering at a fixed wavelength,1,2 while

The authors are with the Centro de Investigaciones Ópticas (CONICET-CIC), Camino Centenario y 506, 1897 La Plata, Argentina. The authors are also with the Área Departamental de Ciencias Básicas, Facultad de Ingeniería, Universidad Nacional de La Plata, Argentina. L. B. Scaffardi is also with the Carrera de Investigador Científico, Consejo Nacional de Investigaciones Científicas y Técnicas (CONICET), Argentina. F. A Videla is also with the Departamento de Electrotecnia, Facultad de Ingeniería, Universidad Nacional de La Plata, Argentina and the Carrera de Profesional de Apoyo, Comision de Investigaciones Científicas y Técnicas de la Provincía de Buenos Aires (CICBA), Argentina. D. C. Schinca (daniels@ciop.unlp.edu.ar) is also with the Carrera de Investigador Científico, Comisión de Investigaciones Científicas y Técnicas (CONICET), Argentina.

Received 18 April 2006; revised 3 August 2006; accepted 25 August 2006; posted 30 August 2006 (Doc. ID 69958); published 15 December 2006.

0003-6935/06/010067-09\$15.00/0

(C) 2007 Optical Society of America others use dynamic light scattering, ${ }^{3}$ working with a fixed angle and a fixed wavelength. Also, some authors addressed the problem of sizing particles in solutions, in laboratory phantoms, and in biological specimens using light scattering spectroscopy methods. ${ }^{4-6}$

In particular, optical backscattering spectroscopy shows advantages over fixed wavelength multiangle scattering measurements. On the one hand, it offers an instrumental advantage, since it may be ensembled endoscopically in a simple and compact way. On the other hand, the rich dependence of the theoretically calculated backscattering coefficient on wavelength and particle diameters can be used for particle sizing.

Previously, ${ }^{4}$ we reported the use of visible backscattering spectroscopy for sizing particles in the 1-24 $\mu \mathrm{m}$ diameter range. Briefly, the analysis was based on the characteristic oscillatinglike nature of the backscattering spectrum when a beam of white light is incident on a suspension of particles, as can be seen in Fig. 1 for a set of calibrated latex particles of $5,8,10$, and $20 \mu \mathrm{m}$ nominal diameters.

In general, the spectra consist of a slowly wavelength-varying part, modulated by a rapidly varying signal. The former represents the combined response of the white lamp spectrum and the sensitivity curve of the detector, while the latter represents the wavelength dependence of the backscattering coefficient. Since this dependence is characteristic for each 


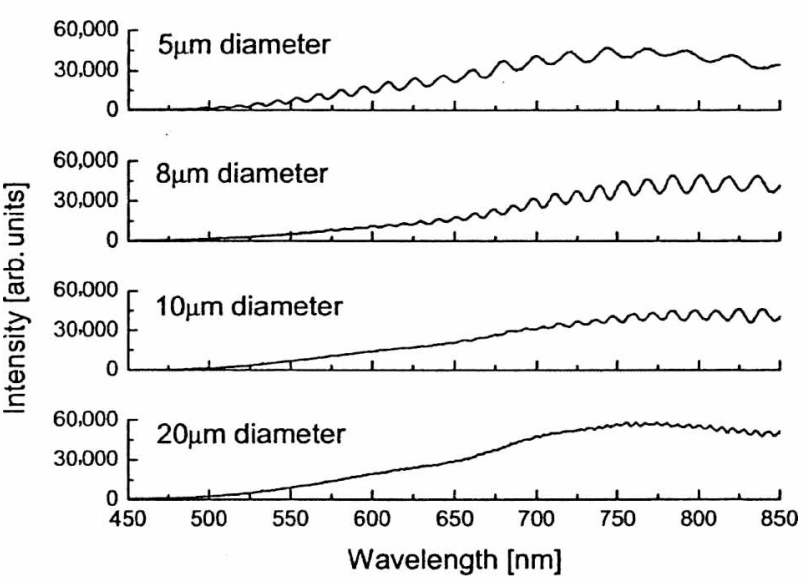

Fig. 1. Experimental raw backscatter spectra for monomodal samples of $5,8,10$, and $20 \mu \mathrm{m}$ latex particle diameters suspended in water.

diameter, it may be used for particle sizing. The number of maxima for a fixed wavelength interval is related to the particle's diameter. Thus if a certain wavelength interval is chosen based on inspection of the backscattering spectra, its frequency content can be determined by Fourier transform (FT) analysis. The oscillating nature of the backscattering signal as a function of wavelength, suggests that its FT should have a relatively sharp maximum whose position should be related to particle diameter. These experimental FTs agree with those obtained from calculated backscattering coefficients using Mie theory and allow for plotting a calibration curve between the FT maximum and the particle diameter. This curve allows for knowing, as a first step, the different particle sizes present in a given sample. Since the FWHM of the FT curve sets a limit on diameter resolution, a second step based on local cross correlation between experimental and theoretical spectra allows for improvement in the accuracy of diameter determination. That work was devoted to sizing particles of diameters between 1 and $24 \mu \mathrm{m}$ using the visible wavelength interval of $600-730 \mathrm{~nm}$. In its conclusions, it was mentioned that the technique could also be used to measure larger particles if the working spectral interval was adequately shifted to NIR wavelengths, based on the observed fact that the oscillations tend to appear more to the $\mathbb{I R}$ as the particle diameter increases. Moreover, it was also mentioned that in this new interval a new calibration curve should be determined according to the procedure described in the cited reference.

Here we extend the application of the backscattering spectroscopy technique to size larger particles and present new experimental backscattering spectra obtained in three NIR wavelength intervals for particles with increasing diameters $(20,30$, and $50 \mu \mathrm{m})$. Our approach basically consists of the application of a simple algorithm to relate the FT maximum of the white-light backscattering spectrum to particle diameter. To increase the accuracy, a second step with cross correlation between experimental and calculated spectra is applied. The FT of experimental signals coming from scatterers have also been successfully applied to fixed wavelength multiangle scattering (FWMS $)^{7}$ for heptane spherical droplets between 10 and $100 \mu \mathrm{m}$. Recently, Semyanov et al. 8 also used FWMS FT to size polystyrene spherical particles ranging from 1.2 to $27.2 \mu \mathrm{m}$ for different refractive index values.

\section{Experimental}

Figure 2 shows the experimental arrangement, which is similar to the one used in a previous work ${ }^{\mathbf{1}}$ : light from a quartz-tunsgten-halogen lamp (visible and NIR) is focused on the cell containing the sample through a delivery optical fiber of $200 \mu \mathrm{m}$ core diameter. The backscattering light is collected by a similar optical fiber (collection) positioned close and parallel to the delivery fiber (separation of the fiber cores between 300 and $500 \mu \mathrm{m}$ ), both immersed in the sample. The collection fiber is connected to an echelle spectrograph multichannel instrument, which is sensitive in the interval of 200-1100 nm (Apogee CCD camera) with a constant resolution of $0.3 \mathrm{~nm}$. The setup of the fibers (distance between fibers and depth when immersed in the sample) leads to a maximum collection cone of approximately $3^{\circ}$ from the backward direction. The wavelength intervals studied in this work cover three NIR regions: $750-850,1060-1100$, and 1110-1150 nm. Standard calibrated monodisperse latex spheres suspended in pure filtered water were used as samples. The nominal calibrated diameters of the particles used were $5,8,10,20,30$, and $50 \mu \mathrm{m}$, traceable to the standard meter through the National Institute of Standards and Technology, as stated by the manufacturer. All the samples were manufactured by Duke Scientific Corporation except for the $1 \mu \mathrm{m}$ diameter particles, which were manufactured by Seradyn. The sample cell was a $10 \mathrm{~cm}$ long and a $1.0 \mathrm{~cm}$ diameter cylinder with an inner flat black coating to avoid wall reflections to reach the collection fiber. The experimental spectra were made free from instrument-dependent features by normalizing to a white reflectance spectrum taken as a reference. Simple scattering was achieved provided that concentration of solids used for the measure-

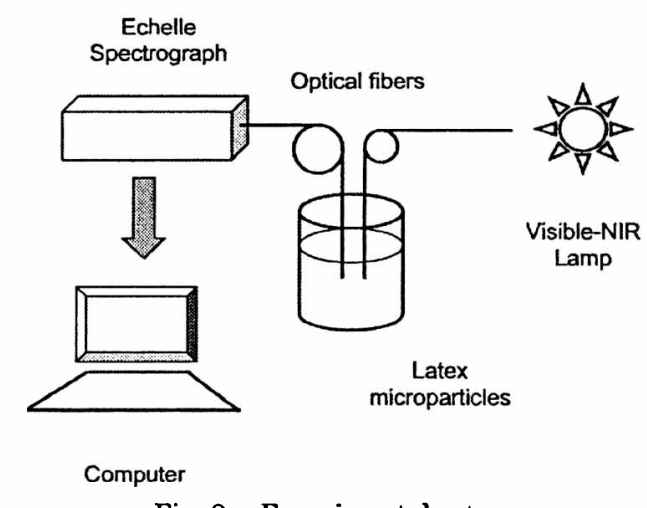

Fig. 2. Experimental setup. 
ments ranged from $10^{-4}$ to $10^{-5} \mathrm{~g} / \mathrm{cm}^{3}$. This condition was always met in our experiments.

\section{Results}

For a better analysis of the spectra, it is convenient to take off the slowly varying component by subtracting an appropriate polynomial that mimics the quartztungsten-halogen spectrum. This procedure is similar to the one used in atmospheric gas pollutant detection using differential optical absorption spectroscopy.9-11 Thus the raw spectra are transformed into accomponent spectra, as seen in Fig. 3(a) for 8, 10, and $20 \mu \mathrm{m}$ particle diameter monodispersed samples in the 750-850 $\mathrm{nm}$ wavelength interval. Two features can be observed: first, the oscillations are almost sinusoidal, and second, the number of maxima (and minima) increases as the diameter increases.

It can be observed in Fig. 1 and in Fig. 3(a) that the period of the oscillations increases with increasing wavelength. This fact is due to the dependence of the scattering coefficient on Bessel and Hankel functions. ${ }^{12}$ When the spectra are plotted versus the wavenumber, an almost constant oscillation period is

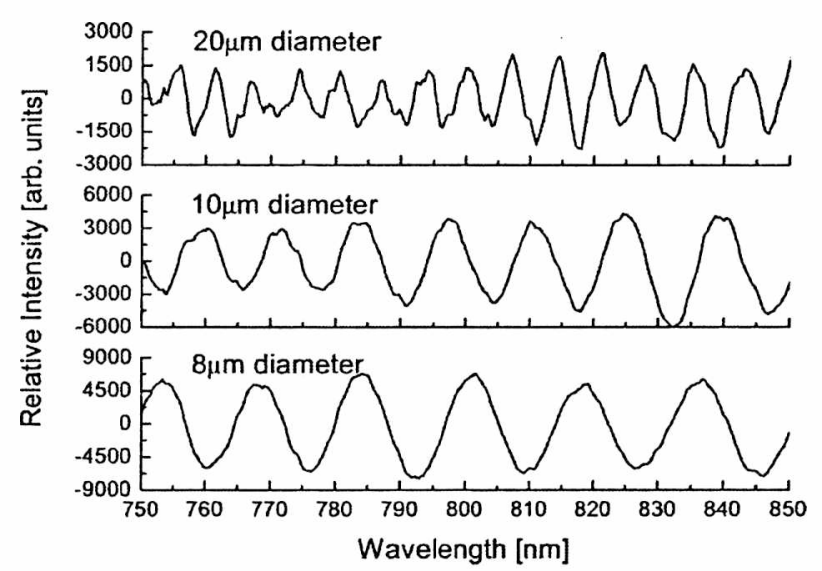

(a)

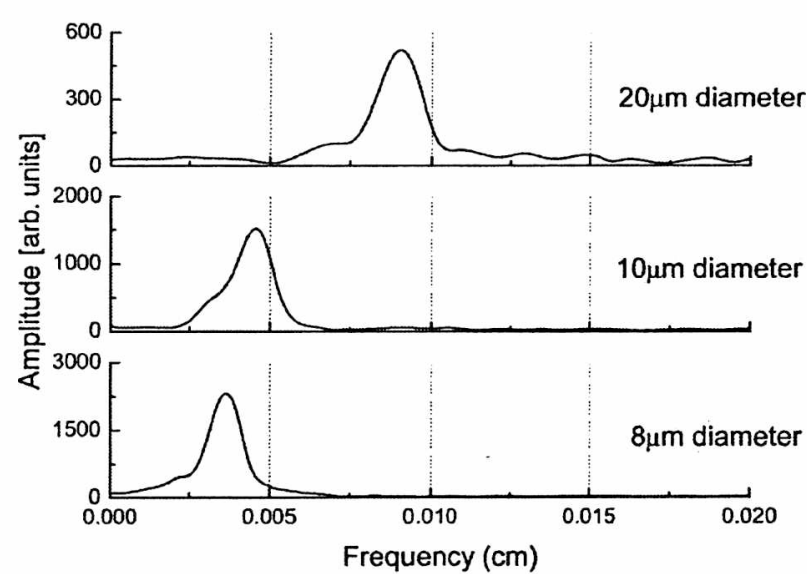

(b)

Fig. 3. (a) Experimental baseline-corrected backscattering spectra for monomodal samples of 8,10 , and $20 \mu \mathrm{m}$ latex particle diameters suspended in water. (b) FT of the spectra of (a). observed, which is more appropriate for FT calculation. All the FT shown in this paper are applied to spectra plotted versus the wavenumber, although, for visualization purposes, the spectra will be shown in the wavelength scale, which is the conventional way that a spectrometer yields information.

In these conditions, if FTs are calculated on the above spectra, two facts can be observed: first, they will show a distinct maximum located at a specific frequency value for each sample, and second, this maximum will shift to larger frequencies as the diameter increases. These behaviors are shown in Fig. 3(b), where the FT of the different spectra shown in Fig. 3(a) (previously plotted as a function of the wavenumber) were calculated with the fast FT algorithm. It can be seen that the curves are almost single peaked, and its position increases monotonically as the particle diameter increases.

For the case of multimodal samples, the different components contribute to the total backscattering intensity, yielding a spectrum that, in general, may not have an almost sinusoidal shape. Figure 4(a) corresponds to the experimental spectrum obtained for a

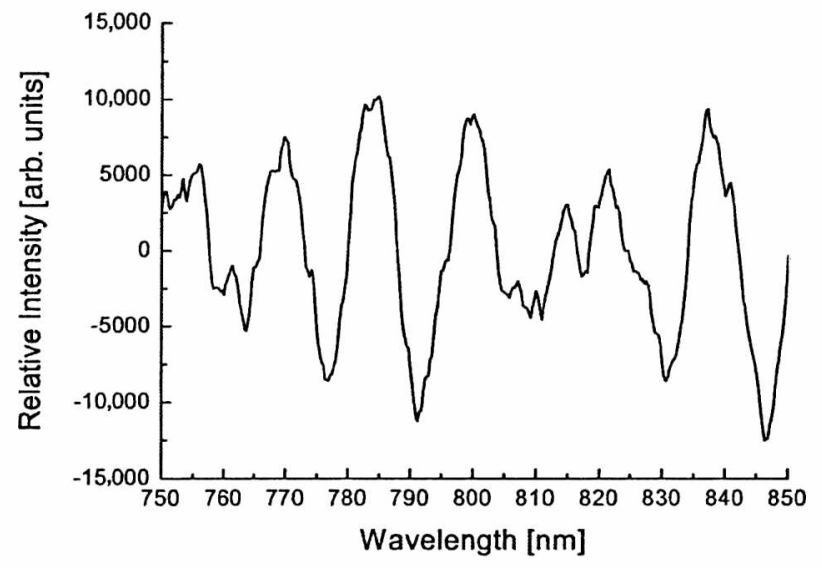

(a)

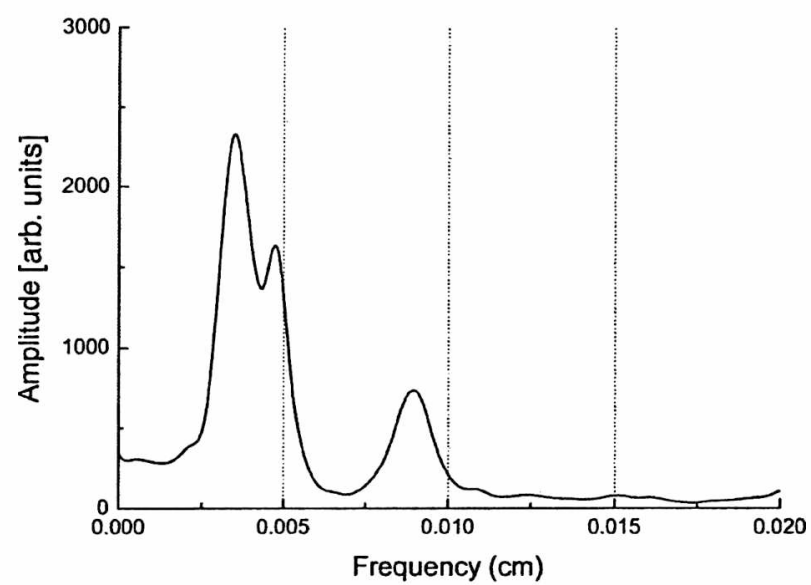

(b)

Fig. 4. (a) Experimental backscattering spectrum for a multimodal sample consisting of a mixture of latex particles of 8,10 , and $20 \mu \mathrm{m}$ diameters suspended in water. (b) FT of the spectra of (a). 


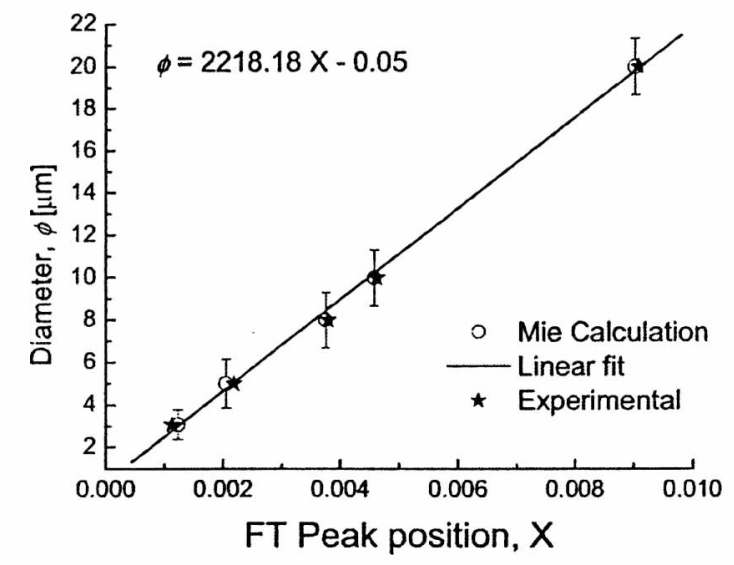

Fig. 5. Relation between the particle diameter and the FT peak position for five diameter values corresponding to spectra in the $750-850 \mathrm{~nm}$ range. Open circles correspond to theoretical calculations. Filled stars correspond to experimental values for the same diameters. The linear fit is on theoretical values.

mixture sample of 8,10 , and $20 \mu \mathrm{m}$ diameter particles. When the FT is applied directly to this backscattering spectrum [Fig. 4(b)], three peaks can be observed, indicating the three sizes present in the sample. Note that the position of the three peaks coincides with the position of the FT maximum for each monomodal spectrum [Fig. 3(b)]. These results are similar to those obtained when studying smaller particles in the $600-730 \mathrm{~nm}$ visible interval. ${ }^{4}$

Nominal diameters $(3,5,8,10$, and $20 \mu \mathrm{m})$ of the particles given by the manufacturer may be plotted versus the maxima of the FT of theoretical backscattering efficiency spectrum (open circles, Fig. 5). It can be seen that the results may be fitted by a linear regression. Filled stars represent FT peaks of experimentally monomodal measured backscattering spectra for the same diameters. Note that they lay on the same linear regression curve. This regression may then be used to determine particle diameter $(\phi)$ from the FT peak position $(X)$. The slope transforms the $X$ value (measured in centimeters) in appropriate units for $\phi$ to be measured in nanometers. The error bars (approximately $\pm 1.3 \mu \mathrm{m}$ ) for each diameter in the figure represent the uncertainty given by the FWHM of the corresponding FT peak. This determination may be considered as a coarse approximation to a more precise value of the particle diameter.

For the improvement of accuracy in an experimental case, diameter determination should consist of a two-step process: first, a coarse value is taken from the FT maximum of the experimental spectrum, and second, a local approximation is taken to a more accurate value applying a cross-correlation algorithm between the experimental spectrum and the calculated ones within the FWHM range of the FT peak. In our case, integral correlation is formally expressed as

$$
C(\varphi)=\int_{\lambda_{\text {low }}}^{\lambda_{\text {bigh }}} I_{\text {exp }}\left(\lambda, d_{\mathrm{FT}}\right) I_{\mathrm{MIE}}\left(\lambda, d_{\mathrm{FT}}+\varphi\right) \mathrm{d} \lambda,
$$

where the limits of the integral correspond to the lowest and highest values of the chosen NIR interval wavelength, $I_{\exp }$ is the experimentally obtained spectrum, $d_{\mathrm{FT}}$ is the value of the diameter calculated by FT (coarse approximation), $I_{\mathrm{MIE}}$ is the spectrum
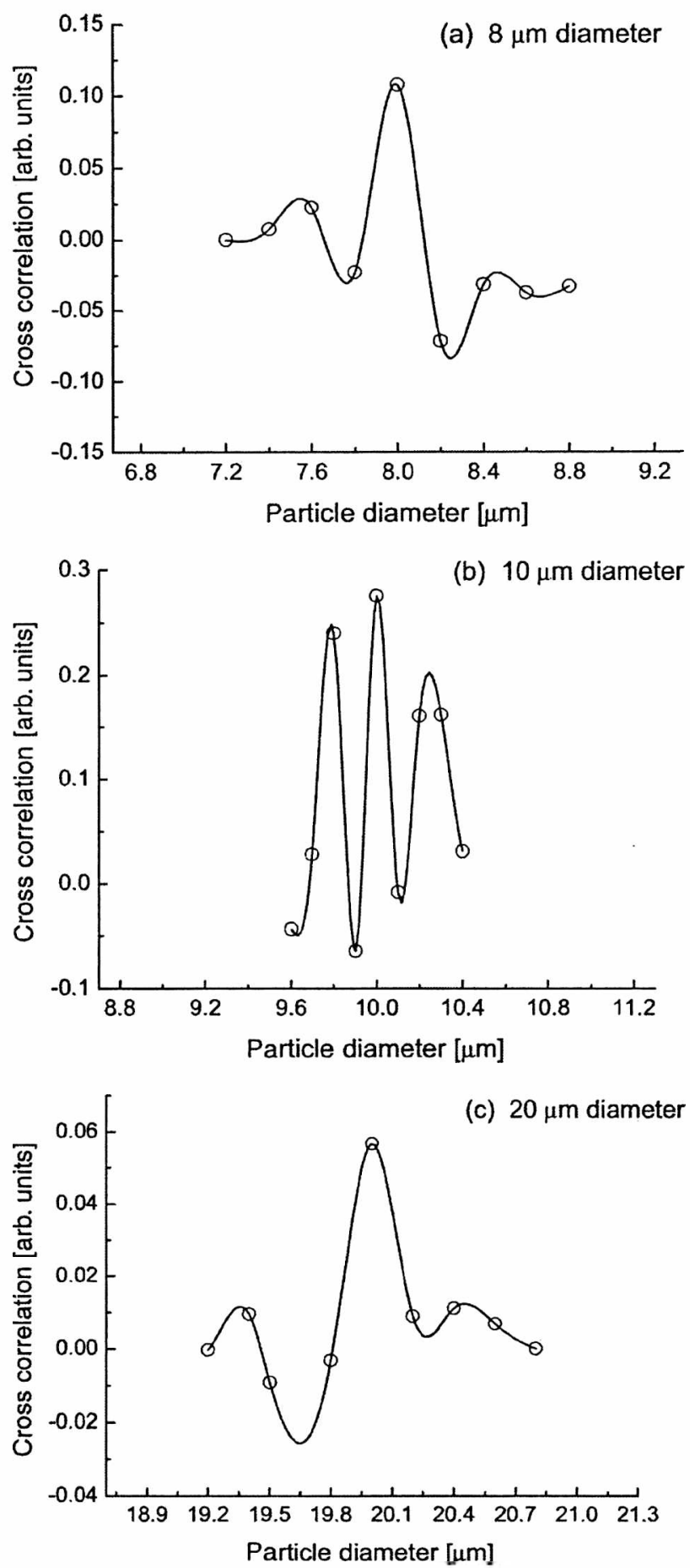

Fig. 6. Cross-correlation results between the mixture spectrum of Fig. 4(a) and calculated monomodal spectra for several diameters at approximately (a) $8 \mu \mathrm{m}$, (b) $10 \mu \mathrm{m}$, and (c) $20 \mu \mathrm{m}$. The span of the horizontal axis corresponds to the uncertainty given by the FWHM of the FT curve. 


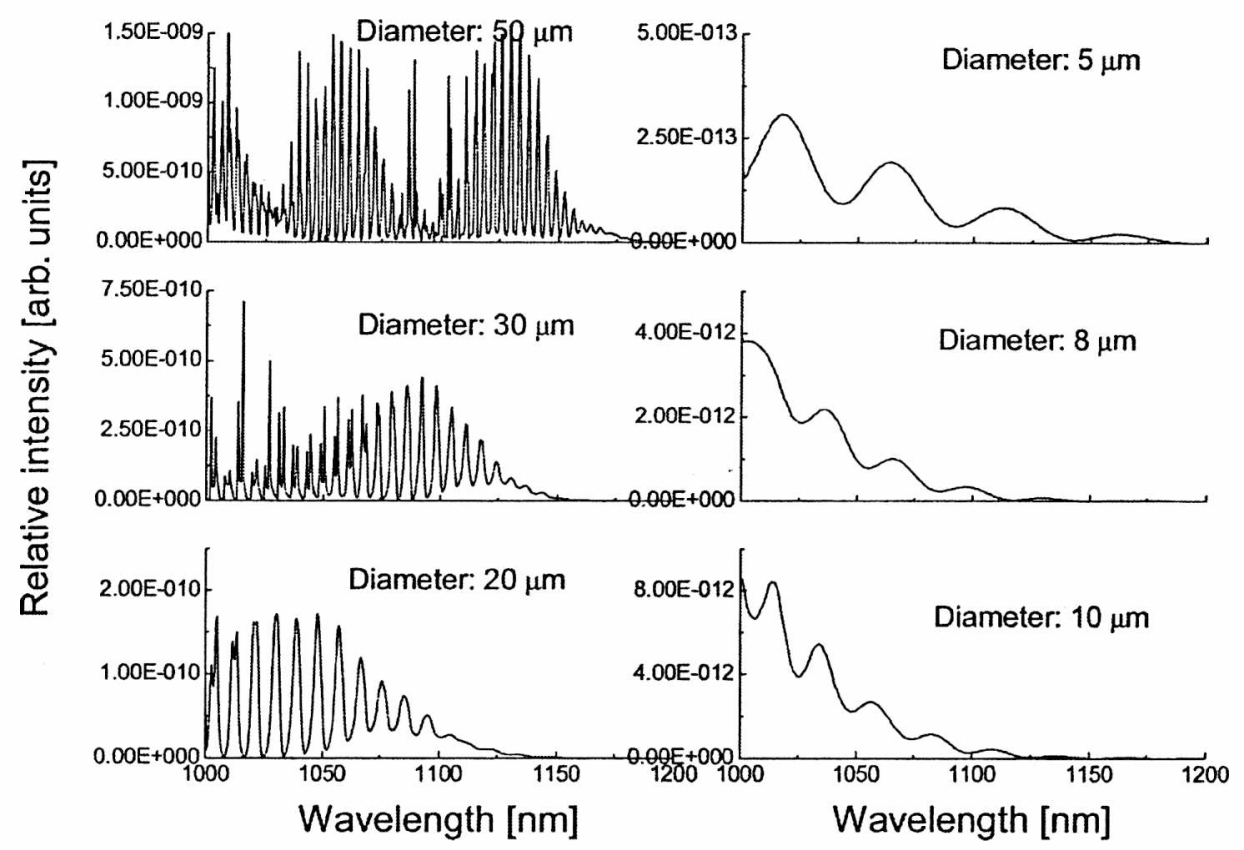

Fig. 7. Calculated backscattering coefficients for different spherical particles as a function of wavelength.

obtained by means of Mie theory, $\lambda$ is the wavelength, and $\varphi$ is the diameter increment around $d_{\mathrm{FT}}$ (delay).

$I_{\mathrm{MIE}}$ for spherical particles is calculated by Mie theory ${ }^{13}$ as a function of wavelength. This calculation is based on the code given by Bohren and Huffman, ${ }^{12}$ where the wavelength dependence of the refractive index of water and particle material was taken into account by ${ }^{1}$

$$
\begin{aligned}
n_{w}(\lambda)= & 1.324+3046 / \lambda^{2} \text { for water, } \\
n_{p}(\lambda)= & 1.59+15 \times 10^{3}\left(1 / \lambda^{2}-1 / 589.32^{2}\right) \\
& \text { for polystyrene particles. }
\end{aligned}
$$

Several $I_{\text {MIE }}$ backscattering spectra are calculated for diameter values at approximately $d_{\mathrm{FT}}$ in an interval covering the FWHM of the FT peak. The largest correlation value for zero delay is then taken as the most probable diameter.

To check the usability of this technique, cross correlation was applied to each peak for the multimodal sample of Fig. 4(a). In this case, $\lambda_{\text {low }}$ and $\lambda_{\text {high }}$ correspond to 750 and $850 \mathrm{~nm}$, repectively. Figure 6 shows these results, where the span of the horizontal axes corresponds to the uncertainty interval derived from the FWHM of the corresponding FT curves. Note that, although the correlation values for each diameter have a sharp absolute maximum within the FWHM range, coincident with the value given by the manufacturer, there are also other lower relative maxima, mainly due to the functional form of the calculated backscattering coefficients in this wavelength interval. This fact settles a criterion for determining the correlation width, that includes these maxima as the new uncertainty in diameter value. It

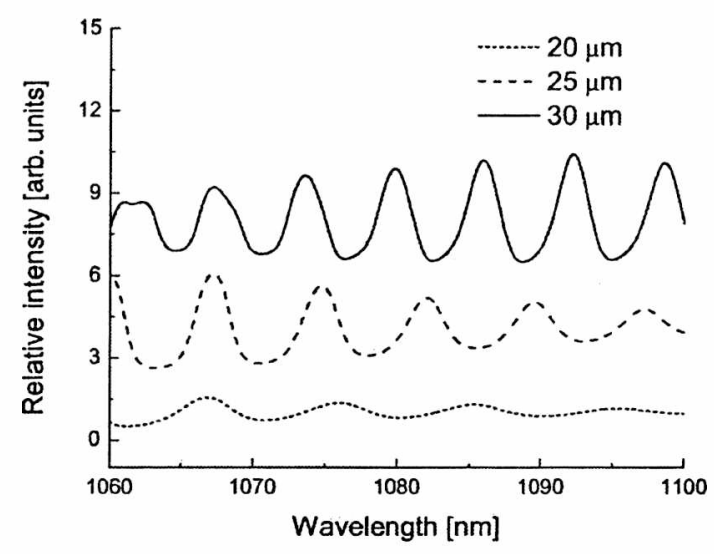

(a)

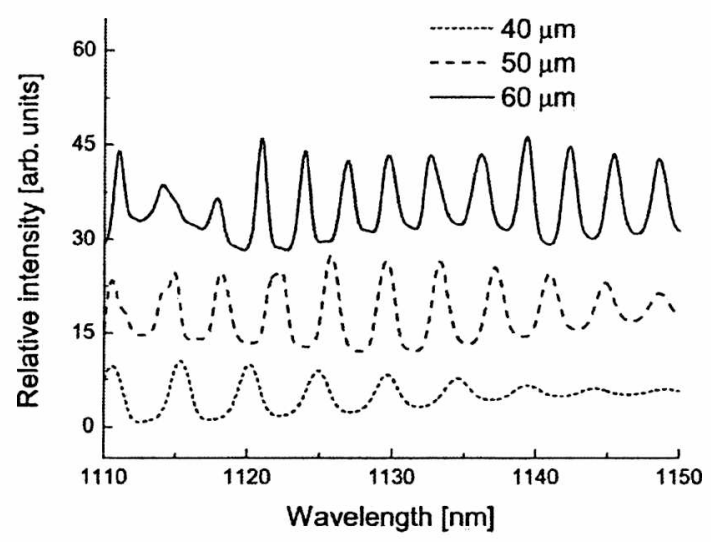

(b)

Fig. 8. Calculated backscattering intensity for (a) 20,25, and $30 \mu \mathrm{m}$ particle diameters between 1060 and $1100 \mathrm{~nm}$ and (b) 40, 50 , and $60 \mu \mathrm{m}$ particle diameters between 1110 and $1150 \mathrm{~nm}$. 
can be observed that this FWHM of the correlation is still narrower than that of the FT. This procedure reduces the uncertainty on the diameter determination in $-35 \%$ in this region.

For larger particles, it is interesting to analyze the spectra of theoretical backscattering coefficients calculated in a wavelength interval that extends farther into the NIR. The backscattering efficiency for spherical particles as a function of wavelength can be calculated using the codes given by Bohren and Huffman ${ }^{12}$ and Laven. ${ }^{14}$ Figure 7 shows an example of such calculations for different sphere diameters $(5,8,10,20$, 30 , and $50 \mu \mathrm{m})$. It can be seen that, as the diameters increase, the backscattering efficiency spectrum extends farther into the NIR. Inspection of the spectra shows that there are wavelength intervals that comprise almost equally spaced oscillation features, typical for each diameter, thus suggesting an almost single-peaked FT spectrum. These wavelength intervals may coincide for a certain range of diameters, but must be changed if larger particle diameters are to be analyzed. In particular, from 20 to $\sim 35 \mu \mathrm{m}$ diameter, a suitable wavelength interval seems to be

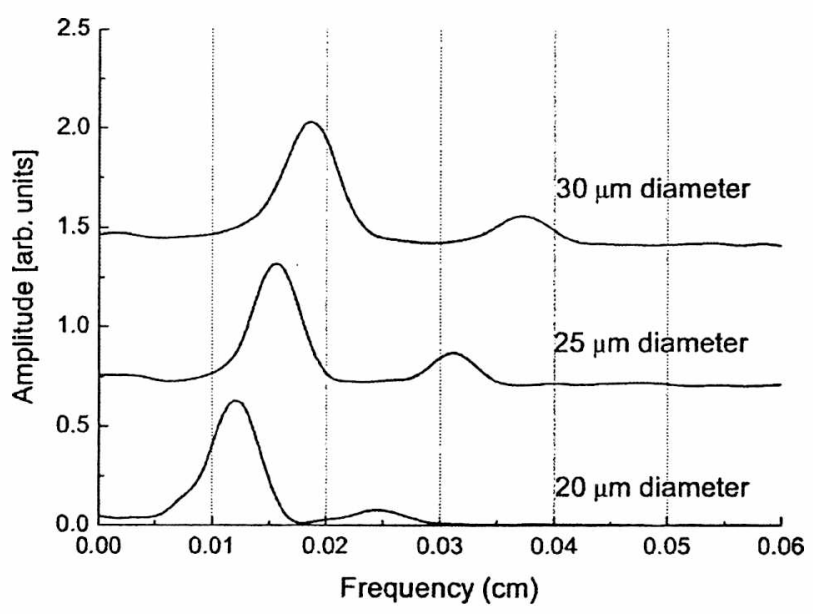

(a)

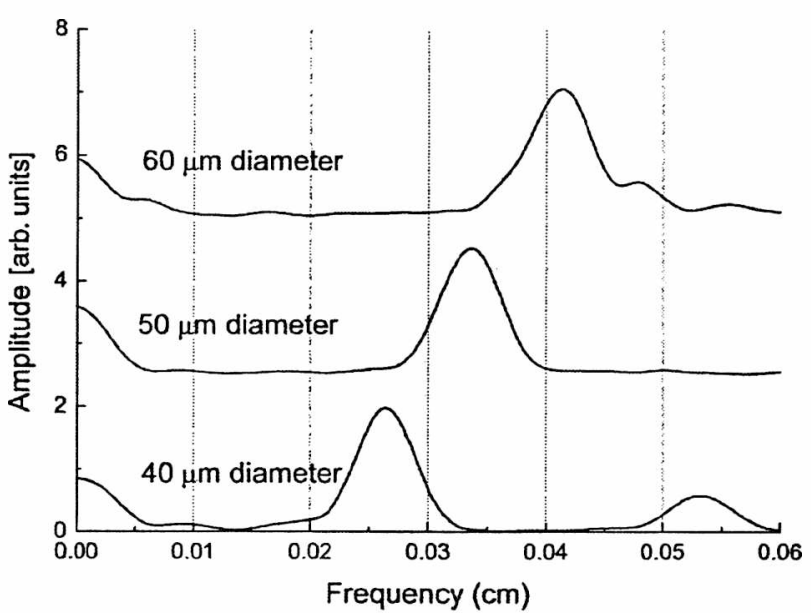

(b)

Fig. 9. FT of spectra of (a) Fig. 8(a) and (b) Fig. 8(b).
1060-1100 nm, while for diameters between 35 and $60 \mu \mathrm{m}$, the most suitable interval is in the range of 1110-1150 nm.

Figure 8 shows the calculated backscattering efficiency spectrum for particle diameters of (a) 20, 25, and $30 \mu \mathrm{m}$ and (b) 40,50 , and $60 \mu \mathrm{m}$. They are grouped into two different wavelength intervals (as stated above), both of the same length. In each group, it can be seen that the curves differ in the number of maxima. When the FT of these spectra (taken as a function of the wavenumber) are calculated, their maxima fall in different positions, as shown in Figs. 9(a) and 9(b). Each FT spectra shows a distinct maximum that shifts to larger values as the particle diameter increases. The secondary maxima account for the slight modulation in the amplitude of the scattering coefficients in the analyzed wavelength interval. Similar to what was shown for the interval of $750-850 \mathrm{~nm}$, the functional relation between the position of the FT peaks and the corresponding particle

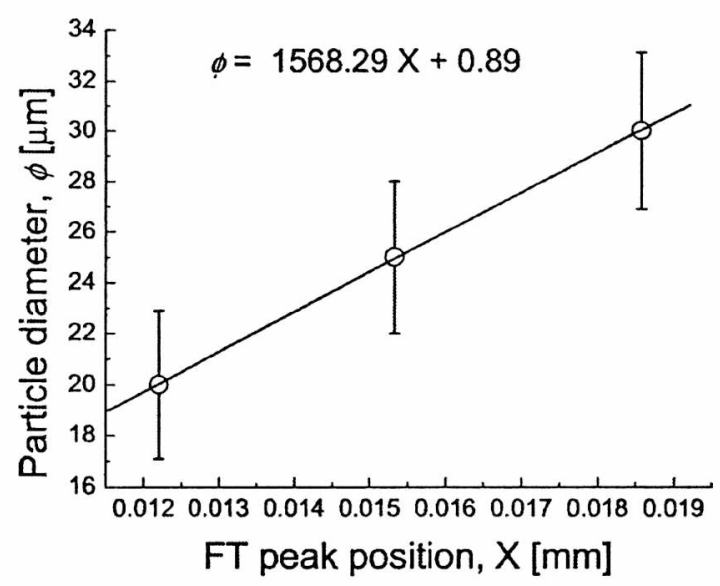

(a)

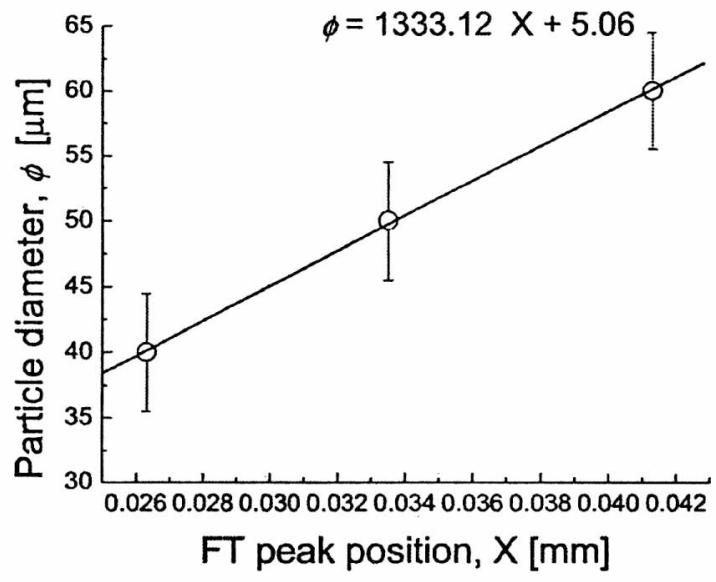

(b)

Fig. 10. Particle diameter versus FT peak position for (a) 20, 25, and $30 \mu \mathrm{m}$ particle diameters (circles with error bars) and linear regression (line) for the 1060-1100 $\mathrm{nm}$ range and (b) 40, 50, and $60 \mu \mathrm{m}$ particle diameters (circles with error bars) and linear regression (line) for the 1110-1150 nm range. The error bars represent the uncertainty given by the FWHM of the FT curve. 
diameter in these new ranges may be fitted by a linear regression for particle diameters between 20 and $35 \mu \mathrm{m}$, and by another linear regression between

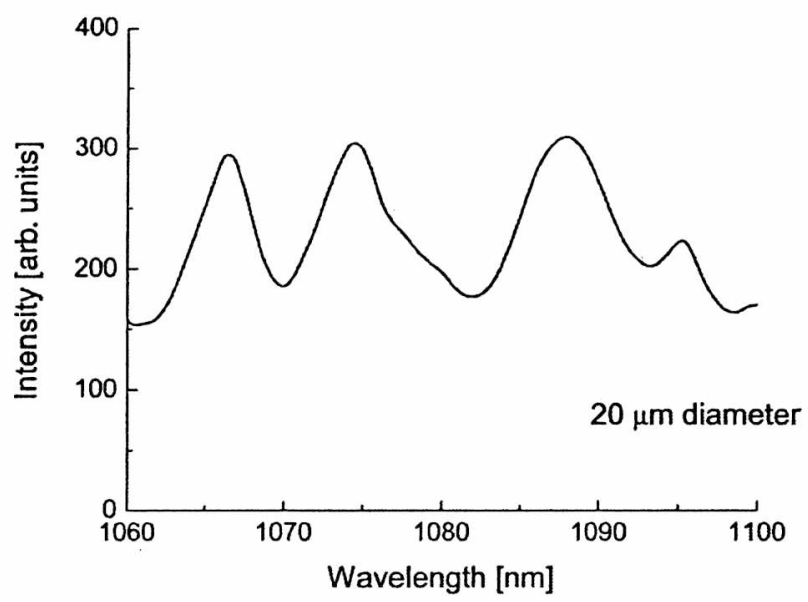

(a)

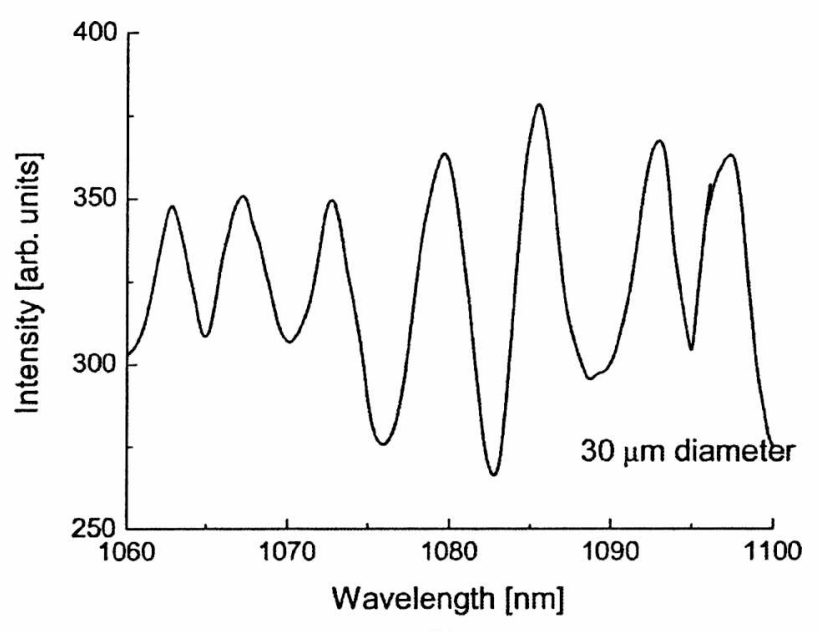

(b)

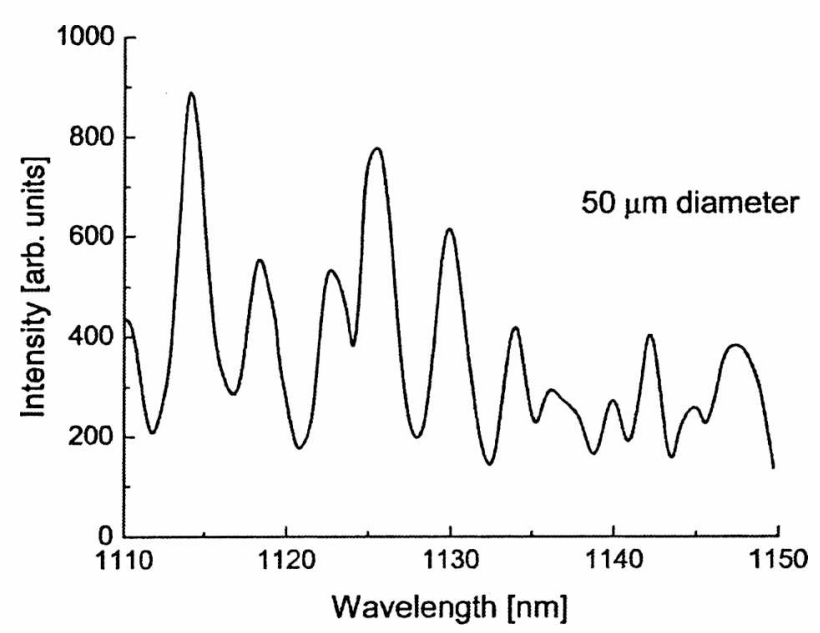

(c)

Fig. 11. Experimental backscattering spectrum. The data were processed according to the guidelines described in the text.

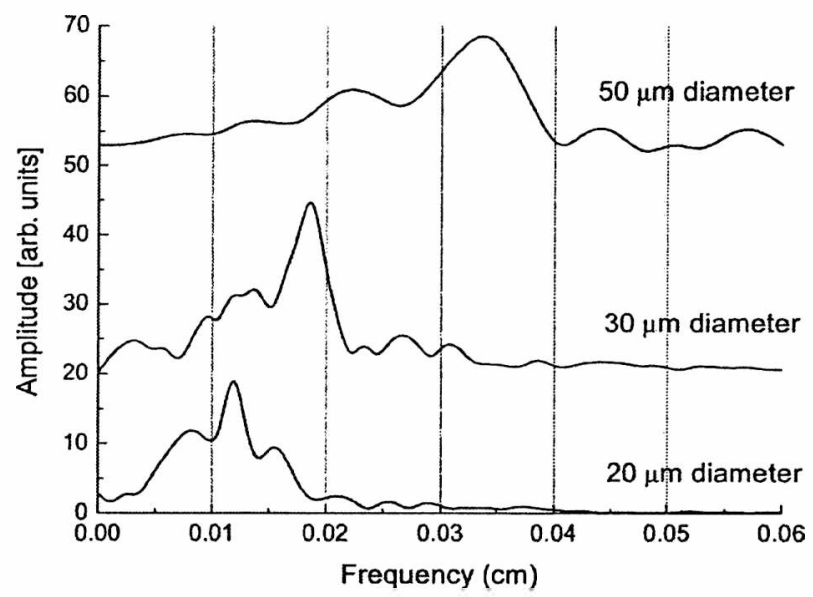

Fig. 12. Stacked FT of the experimental spectra of Fig. 11.

35 and $60 \mu \mathrm{m}$, each with its own slope and error bars given by the FWHM of the corresponding FT peaks. Such curves are depicted in Figs. 10(a) and 10(b) for the wavelength intervals of $1060-1100 \mathrm{~nm}$ and 1110-1150 nm, respectively. To test the method in these two NIR intervals, we recorded experimental backscattering spectra for samples of 20,30 , and $50 \mu \mathrm{m}$ nominal diameter particles, using the instrumental setup described previously. The selected wavelength intervals coincide with those shown in Figs. 8(a) and 8(b). Unfortunately, these wavelengths are at the edge of the sensitivity curve of our detector (silicon CCD), where the signal-to-noise ratio is very poor.

To enhance the useful signal, the CCD background noise was first subtracted from the raw backscattering spectra and then displayed as a function of the wavenumber. The remaining highfrequency noise was almost removed by smoothing with a seven-point moving average and then cor-

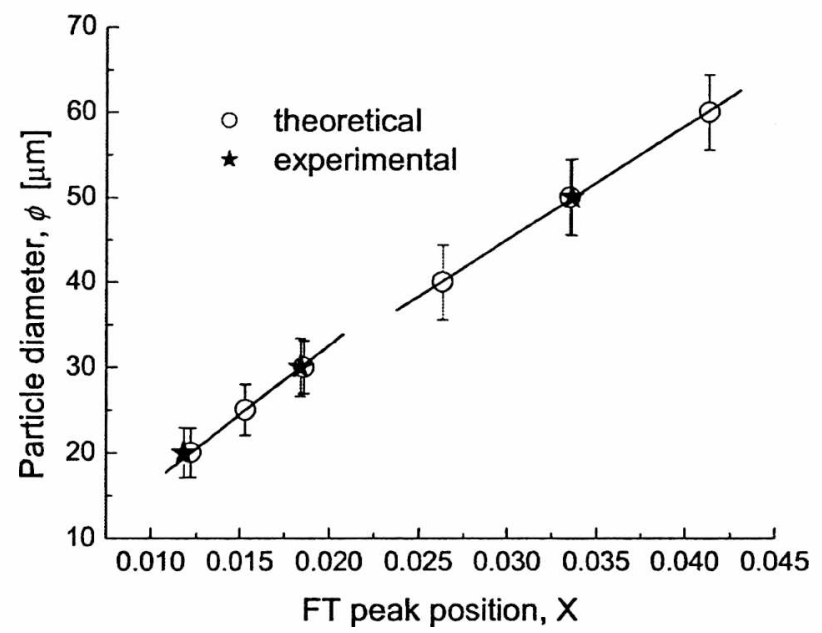

Fig. 13. Comparison of the particle diameter versus the FT peak position relation for calculated $20-60 \mu \mathrm{m}$ particle diameters (circles with error bars) and experimental 20,30 , and $50 \mu \mathrm{m}$ particle diameters. 


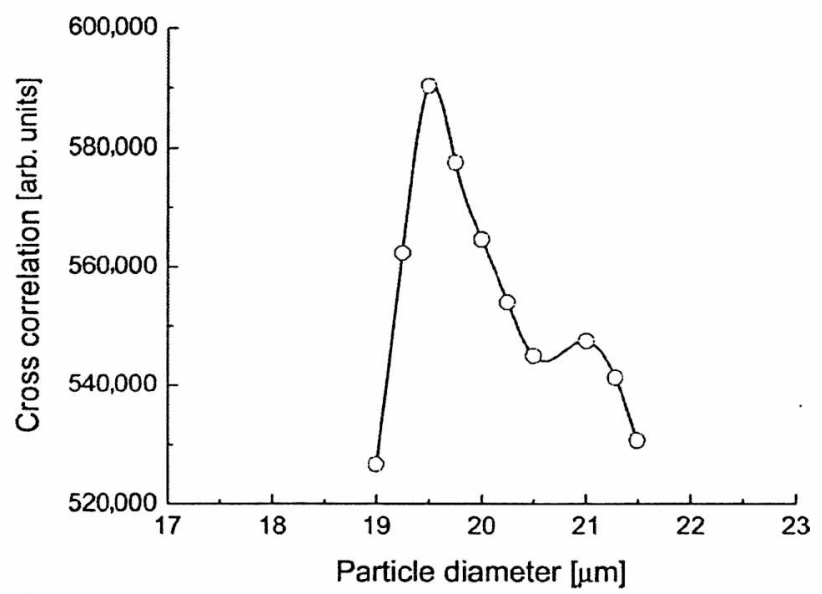

Fig. 14. Correlation values for backscattering spectrum corresponding to a $20 \mu \mathrm{m}$ nominal diameter. The span of the horizontal axis corresponds to the uncertainty interval derived from the FWHM of the FT curve.

rected by subtracting a suitable slowly varying baseline. At this step, the signal is almost free from its dc component and only rapidly wavelength varying ac components are present, which can be explored using the FT. In fact, all the FT obtained in this work were applied to signals processed in this way. Figures 11(a)-11(c) show the obtained spectra for 20,30 , and $50 \mu \mathrm{m}$ particle diameters, respectively. It can be seen that, in general, the sequence of peaks and valleys correspond to those calculated for the backscattering efficiency coefficient depicted in Fig. 7, although the whole signal seems to be modulated by a slowly varying noise.

Figure 12 shows the stacked FT for the three experimental backscattering spectra of Fig. 11. Note that, in spite of the noisy signals, the FT spectra still show distinct peaks that agree well with those corresponding to theoretical calculations.

These peak values (with their corresponding error bars) are shown in Fig. 13, together with the FT peak values derived from the Mie calculations, taken from
Fig. 10. It can be seen that the experimental values lay well within each linear fit.

Again, the observed linear relation between the FT maxima and the particle diameter may be used as a first approach to determine the diameter of particles in a sample at these wavelength intervals. Following the previously described procedure, at this point the cross-correlation technique may be applied. Results for $20 \mu \mathrm{m}$ nominal diameter particles are shown in Fig. 14 for the wavelength interval of $1060-1100 \mathrm{~nm}$. The span of the horizontal axis has been set equal to the error given by the FWHM of the corresponding FT. It can be observed that a correlation maximum appears for a certain diameter value, very close to that obtained by the FT. The uncertainty width reduces to $\sim 33 \%$ of that corresponding to the FT. However, this reduction is not as good as in the case of smaller particles (1-20 $\mu \mathrm{m}$ diameter) analyzed in the visible range. ${ }^{4}$

Thus the calibration curves obtained for the three analyzed spectral intervals allow, in general, for the solving of any multimodal sample having particles between a 3 and $60 \mu \mathrm{m}$ diameter. If the sample contains a mixture of small, medium, and large particles, they will contribute to different spectral intervals. The FT calculated for each interval will yield peaks corresponding to the different particles present in the sample which, in turn, can be explored using the correlation step.

As mentioned in Section 1, other authors ${ }^{6}$ analyzed small biological particles (hundreds of nanometers in diameter) using light scattering spectroscopy. They worked with least-squares techniques for size distribution determination and achieved a reduction in uncertainty compatible with the diameter range studied. In the case of larger particles, as the ones considered in this work (tens of micrometers), the FT yields a satisfactory first approach to size determination in multimodal samples. A finer approach using cross correlation reduces the uncertainty by $\sim 30 \%$, which is acceptable for this diameter range.

Finally, considering the results obtained in the previous work for the visible $600-730 \mathrm{~nm}$ region, ${ }^{4}$ there

Table 1. Spectral Intervals and Particle Diameters Analyzed with Backscattering Spectroscopy Using FT and Cross Correlation

\begin{tabular}{|c|c|c|c|c|}
\hline $\begin{array}{l}\text { Spectral Range } \\
\text { of Analysis } \\
\text { (nm) }\left[\lambda_{\text {low }}, \lambda_{\text {high }}\right]\end{array}$ & $\begin{array}{l}\text { Range of } \\
\text { Diameters } \\
{\left[\phi_{i}, \phi_{r}\right]} \\
{[\mu \mathrm{m}]}\end{array}$ & $\begin{array}{c}\text { FT Linear Regression } \\
X=\text { FT Peak Position } \\
\text { [cm] } \phi=\text { Diameter } \\
{[\mu \mathrm{m}]}\end{array}$ & $\begin{array}{c}\text { Uncertainty in FT } \\
\text { Linear Regression } \\
{[\mu \mathrm{m}]}\end{array}$ & $\begin{array}{c}\text { Mean Uncertainty } \\
\text { after Cross } \\
\text { Correlation } \\
{[\mu \mathrm{m}]}\end{array}$ \\
\hline$[600,730]^{b}$ & {$[0.5,10]$} & $\phi=2340.11 X+0.04$ & \pm 0.7 & $\begin{array}{c} \pm 0.025 \\
( \pm 0.030)\end{array}$ \\
\hline$[750,850]^{c}$ & {$[10,20]$} & $\phi=2218.18 X-0.05$ & \pm 1.3 & $\begin{array}{c} \pm 0.50 \\
( \pm 0.10)\end{array}$ \\
\hline$[1060,1100]^{c}$ & {$[20,35]$} & $\phi=1568.29 X+0.89$ & \pm 3 & $\begin{array}{l} \pm 1 \\
( \pm 0.16)\end{array}$ \\
\hline$[1110,1150]^{c}$ & {$[35,60]$} & $\phi=1333.12 X+5.06$ & \pm 4.4 & $\begin{array}{c} \pm 1.47 \\
( \pm 0.51)\end{array}$ \\
\hline
\end{tabular}

'Results in the first row are from a previous work.

${ }^{b}$ Reference 4.

'This work. 
are four wavelength intervals that can be used for particle sizing with backscattering spectroscopy in the visible and NIR.

Table 1 summarizes the overall results. Column 1 shows the spectral interval of analysis. Column 2 shows the diameter range that is best measured in the corresponding spectral interval (linear piecewise representation). Column 3 shows the linear regressions corresponding to the different wavelength intervals analyzed, which allow for calculation of the diameter of the particles in a first approach, together with the corresponding uncertainties. Column 4 shows the mean uncertainty due to FT linear regression. Column 5 shows the mean uncertainties after applying cross correlation. Both mean uncertainties represent the average errors for the different diameter values within the specified range. The specific uncertainty shows an increasing trend as the diameter increases. In this last column, the mean uncertainties given by the manufacturer are shown in parentheses for comparison purposes.

\section{Conclusions}

We have shown that it is possible to size microparticles in the range of $20-60 \mu \mathrm{m}$ diameters in a reliable and simple way using NIR backscattering spectroscopy. It has been demonstrated that the FT of the scattering spectra has a characteristic maximum, whose position is linearly related to the particle's diameter within a specific wavelength interval. A first approximation to particle diameter may be obtained through this linear relation. To improve determination accuracy, a cross-correlation technique between calculated and experimental spectra was applied. This improvement is range dependent, thus forcing a trade-off between sizing capability and accuracy. Together with the results obtained in a previous work on visible backscattering spectroscopy, the improvement due to correlation is very good for a $0.5-10 \mu \mathrm{m}$ particle diameter in the visible interval (3.5\%), good for a 10-20 $\mu \mathrm{m}$ particle diameter in the 750-850 $\mathrm{nm}$ interval (38\%), and acceptable for a 20-60 $\mu \mathrm{m}$ particle diameter in the $1060-1100 \mathrm{~nm}$ and 1110-1150 nm intervals (33\%). Overall, the method yields reasonable accuracy when compared with that of the manufacturer. This method could be used to construct a portable and practical measurement instrument. The possible disadvantages of measuring in the NIR range beyond $1000 \mathrm{~nm}$ with a CCD sensor may be overcome by using a NIR sensitive array such as, for example, InSb.

This work was partially supported by research project 11/I104 from Facultad de Ingeniería, Universidad Nacional de La Plata. This work is the basis of a pending patent, P050101083, INPI, Argentina.

\section{References}

1. L. B. Scaffardi, J. O. Tocho, L. L. Yebrin, and C. S. Cantera, "Sizing particles used in the leather industry by light scattering," Opt. Eng. 35, 52-56 (1996).

2. G. Gouesbet and G. Gréhan, eds., Optical Particle Sizing (Plenum, 1988).

3. B. Chu, Laser Light Scattering: Basic Principles and Practice (Academic, 1991)

4. F. Videla, D. Schinca, and L. B. Scaffardi, "Sizing particles by backscattering spectroscopy and Fourier análisis," Opt. Eng. 45, 048001 (2006).

5. J. Mourant, T. Fuselier, J. Boyer, T. Johnson, and I. Bigio, "Predictions and measurements of scattering and absorption over broad wavelength ranges in tissue phantoms," Appl. Opt. 36, 949-957 (1997).

6. H. Fang, M. Ollero, E. Vitkin, L. M. Kimerer, P. B. Cipolloni, M. M. Zaman, S. D. Freedman, I. J. Bigio, I. Itzkan, E. B. Hanlon, and L. T. Perelman, "Noninvasive sizing of subcellular organelles with light scattering spectroscopy," IEEE J. Sel. Top. Quantum Electron. 9, 267-276 (2003).

7. S. Min and A Gomez, "High-resolution size measurements of single spherical particles with a fast Fourier transform of the angular scattering intensity," Appl. Opt. 35, 4919-4926 (1996).

8. K. Semyanov, P. Tarasov, A. Zharinov, A Chernyshev, A Hoekstra, and V. Maltsev, "Single-particle sizing from light scattering by spectral decomposition," Appl. Opt. 43, 51105115 (2004).

9. D. Perner and U. Platt, "Detection of nitrous acid in the atmosphere by differential optical absorption," Geophys. Res. Lett. 6, 917-920 (1979).

10. H. Edner, P. Ragnarson, S. Spannare, and S. Svanberg, "Differential optical absorption spectroscopy (DOAS) system for urban atmospheric pollution monitoring," Appl. Opt. 32, 327-333 (1993).

11. F. A. Videla, D. C. Schinca, and J. O. Tocho, "Alternative method for concentration retrieval in differential optical absorption spectroscopy for atmospheric-gas pollutant measurements," Appl. Opt. 42, 3653-3661 (2003).

12. C. F. Bohren and D. R. Huffman, Absorption and Scattering of Light by Small Particles (Wiley, 1983).

13. G. Mie, "Beitrage zur Optik trüber Medien speziell kolloidaler Metallösungen," Ann. Phys. 25, 317-445 (1908).

14. P. Laven, MiePlot, 2005, http://www.philiplaven.com/ mieplot.htm. 